\title{
Gopher FITStep Pro accuracy when measuring steps and moderate-to-vigorous physical activity
}

\author{
Ali Brian",* and Justin A. Haegele ${ }^{2}$ \\ ${ }^{1}$ Department of Physical Education, University of South Carolina, Columbia, SC, USA; and ${ }^{2}$ Department of Human Move- \\ ment Sciences, Old Dominion University, Norfolk, VA, USA
}

Copyright: (c) 2017 A. Brian and J. A. Haegele. This is an open access article licensed under the Creative Commons Attribution License (http://creativecommons.org/licenses/by/4.0/).

\begin{abstract}
Background: The Gopher FITStep Pro (GFSP) is a commercially available objective physical activity monitor that records steps taken and moderate-to-vigorous physical activity (MVPA). Objective: The purpose of this study was to evaluate the accuracy of the GFSP for measuring steps taken in a guided walking condition and MVPA during planned fitness activities. Method: University-aged participants ( $\left.N=35, M_{\text {age }}=20\right)$ wore two GFSP (right and left side) pedometers and one ActiGraph GT3X+ accelerometer during both conditions. Results: Paired samples $t$-tests determined that self-step counts in the guided walking condition were not significantly different than the right side GFSP $(p=.084)$ but were significantly different from the left side GFSP $(p=.006)$. Mean absolute percent error (MAPE) was less than $3 \%$ between self-step counts with the left (1.9\%) and right side GFPS (1.7\%). However, MVPA estimates were significantly different between the GT3X+ accelerometer and the left side and right side GFSP $(p<.001)$. High MAPE occurred between the GT3X+ accelerometer with the left (51\%) and right side GFSP (41\%) in the planned fitness activity condition. Conclusion: The GFSP may be an appropriate instrument for estimating steps, however users should be cautious when consuming MVPA estimates for educational, research, or health-related purposes.
\end{abstract}

Keywords: pedometer, accelerometer, validation, physical education

\section{Introduction}

As a result of the growing concern about obesity and the importance of physical activity, new exercise programs and products are gaining popularity internationally. For example, commercially available, wearable, objective physical activity monitors are widely used as personal devices, as well as for school-based physical education programming. Two physical activity monitors that are widely used for individuals of all ages are pedometers and accelerometers (Lee, Williams, Brown, \& Laurson, 2015). Contemporary pedometers are small devices that are usually worn on the hip (Lee et al., 2015) and are relatively low in cost (Clemes \& Biddle, 2013). Typically, low-cost pedometers evaluate physical activity using a spring-loaded mass to detect the obvious impacts produced by steps during locomotion (Yang \& Hsu, 2010). Spring-loaded technology relies on a horizontal spring-suspended pendulum arm

\footnotetext{
* Address for correspondence: Ali Brian, Department of Physical Education, University of South Carolina, 1300 Wheat St., 29208, Columbia, SC, USA. E-mail: brianali@mailbox.sc.edu
}

that moves up and down with each step and opens and closes an electrical circuit (Steeves et al., 2011). Unfortunately, however, spring-loaded pedometers are susceptible to errors, particularly with overweight/ obese users (Steeves et al., 2011). More specifically, as tilt angle increases (as a result of increases in the users' body mass index), accuracy decreases (e.g., Hasson, Haller, Pober, Staudenmayer, \& Freedson, 2009). Furthermore, pedometers using this technology are limited in that they cannot identify or estimate physical activity intensity (Yang \& Hsu, 2010).

Accelerometers, on the other hand, provide a more robust and detailed physical activity evaluation for users by measuring the accelerations of objects in motion along reference axes (Gatti, Stratford, Brenneman, \& Maly, 2015). This technology estimates the intensity and duration of physical activity participation for users and accelerometers are widely used in physical activity and exercise research across populations (e.g., Elies, 2015; Gatti et al., 2015; Lee et al., 2015). Recent advances in technology have reduced the cost of motion-sensing technology associated with previously high-priced accelerometers (Yang \& Hsu, 2010). 
A number of pedometers are now using motion-sensing technology rather than the traditional spring-loaded mass. Research has indicated that several pedometers (i.e., Omron HJ-720ITC) utilizing motion-sensing technology have superior accuracy when compared to traditional spring-levered pedometers (Crouter, Schneider, \& Bassett, 2005; Hasson et al., 2009).

Pedometers and accelerometers have long been considered important tools for understanding physical activity patterns (e.g., Brusseau et al., 2011) or introducing interventions (e.g., Kurti \& Dallery, 2013) among youth in school settings (Brusseau \& Burns, 2013). In recent years, the utilization of objective physical activity monitors has also been highlighted as a means of promoting physical activity during physical education classes (Brusseau \& Burns, 2015). As such, entire activity guides have been developed where keeping track of physical activity using objective monitors is embedded into activities (Pangrazi, Beighle, \& Sidman, 2003). Thus, pedometers are now being tailored to the needs of and marketed toward physical educators. The Gopher FITStep Pro (GFSP; Gopher, 2015) is one such pedometer is heavily marketed toward physical educators for use in school-based settings. As such, it includes a number of convenient functions for physical educators, such as a large display screen and printable activity scores that are easy to understand. When using physical activity monitors, obtaining valid and accurate measurement is essential (Barreira et al., 2013). As such, obtaining an accurate assessment has become a critical component of obesity and physical activity research (Wetten, Batterham, Tan, \& Tapsell, 2014). If the GFSP can accurately measure moderateto-vigorous physical activity (MVPA), it may be a feasible instrument to use in school-based programs (e.g., physical education). In addition to its potential use in physical education classes, the GFSP may be practical for large-scale research assessing physical activity. To the knowledge of the authors, the GFSP pedometer has not been thoroughly evaluated to determine the accuracy of steps taken or MVPA. Therefore, the purpose of this study was to evaluate the accuracy of the GFSP for measuring (a) steps taken in a guided walking condition and (b) MVPA in planned fitness activities within a university physical fitness course.

\section{Methods}

\section{Participants and setting}

Upon receiving approval from the institutional review board at the University of South Carolina, volunteers were solicited for participation from a physical fitness course. Thirty-five university-aged students $\left(M_{\text {age }}=20\right.$; women $=12$, men $=23$; African-American $=5$; Caucasian $=30$ ) agreed to serve as participants and provided written consent. Impaired ambulation was the only exclusion criterion applied to participant sampling. No student reported having a documented disability. This study took place on the campus of a southern university in a physical activity and recreation building. Step count training occurred within a classroom where the training video was projected onto a large screen. Data collection was conducted in guided walking and a planned fitness activities conditions. These conditions occurred during a university physical fitness course held in a gymnasium, which included two full-court basketball courts.

\section{Instruments}

\section{Pedometer}

The GFSP (Gopher, Ovatonna, MN, USA) was the pedometer used for this study. According to Gopher (2015), the GFSP features a mechanism that has been described as being accurate even when the pedometer is not perfectly upright, as well as a delayed-counting feature that measures true activity time by preventing inflated step totals by only counting consecutive steps taken after activity begins. The GFSP also includes a large, easy-to-read display that features MVPA outputs. This product is specifically designed and promoted for utilization in physical education classes, and includes a function to upload and organize data into printable reports for teachers, students, and parents. All users determined their walking steps per minute (between 80 to 150) after walking comfortably for one minute. Each user inputted their steps into the GFSP rounding to the nearest ten (e.g., 57 became 60).

\section{Accelerometer}

The GT3X+ ActiGraph accelerometer (ActiGraph, Fort Walton Beach, FL, USA) acted as the MVPA criterion measure for the planned fitness activities. The $\mathrm{GT} 3 \mathrm{X}+$ is a triaxial accelerometer, which is commonly used in physical activity research and is considered a valid and reliable measure of MVPA (Jarrett, Fitzgerald, $\&$ Routen, 2015). All accelerometers were initialized to record data in $80 \mathrm{~Hz}$ using ActiLife 6 software version 6.1 and used the low-frequency extension filter. Analyses were set to a 10-second epoch and featured adult cut points by Freedson, Melanson, and Sirard (1998):

- sedentary: 0-99 counts per minute (CPM),

- light: 100-1,951 CPM,

- moderate: 1,952-5,724 CPM,

- vigorous: 5,725-9,498 CPM,

- very vigorous: 9,499+ CPM. 


\section{Self-step counts}

Self-step counts were utilized as the criterion measure for the guided walking condition. Prior to participating in the study, participants completed a self-step count training protocol. Self-step counts were utilized during the closed-circuit condition only.

\section{Procedures}

There were three phases within this study: (a) self-step count training protocol, (b) guided walking, and (c) a planned fitness activities condition. Guided walking protocols allow for researchers to collect data during short walking bouts with a pre-selected walking pace (Pitchford \& Yun, 2011). On the other hand, the planned fitness activities phase asked participants to maintain individualized physical activity patterns at a moderate-to-vigorous intensity throughout the duration of the measurement time.

\section{Training protocol}

Once enrolled in the study, participants completed a step-count training protocol. The step-count training protocol started with participants viewing a two-minute video of an adult female walking a controlled course at a pace of 110 steps per minute. A total of 220 steps occurred during the training video. Participants viewed the video on an overhead projector in a large classroom, individually tallied the number of steps of the adult woman walking in the video, and recorded the total steps on a record sheet. Participants continued the training protocol until they successfully counted steps within two percent of the actual step count. All participants successfully completed the training protocol.

\section{Guided walking}

Participants were enrolled into the guided walking phase of the study after successfully completing the training protocol. Participants wore an elastic accelerometer belt on which physical activity devices were mounted. Three devices were mounted on the belt for each participant, including one GT3X+ ActiGraph accelerometer (located on the right iliac crest above the midaxillary line) and two GFSP pedometers (placed over the anterior midline of the left and right thigh). Prior to data collection, participants completed a selfcounted 20-step test comparing self-counts with both right and left pedometers to ensure proper placement and counting accuracy (Tudor-Locke \& Myers, 2001).

After successful completion of the 20-step test and any necessary placement modifications, participants completed the one minute self-selected walking pace to set the steps per minute ratio needed for the GFSP to calculate MVPA. Next, participants walked a twominute closed course with the display screen for each
GFSP covered. The closed course was similar to that of Pitchford and Yun (2011) where participants followed a pattern with both left and right turns for a total distance traveled of 223 feet. Each diagonal pathway was 33 feet (Figure 1). Participants self-counted steps during the two-minute trial and carried a recording sheet to $\log$ the results of the two-minute course immediately at the end of the two minutes. Subsequently, participants recorded self-counts and then uncovered the display screens for both GFSP devices to record step counts for both devices.

\section{Planned fitness activities.}

The last phase of the study included measuring MVPA within planned fitness activities during a university physical activity course. Participants started the planned fitness activities portion of the physical fitness course after the GFSPs were set. A timeframe of 25 minutes was allotted for the planned fitness activity phase. During the planned fitness activities, participants chose to participate in either basketball $(n=18)$ or soccer games/activities $(n=17)$. Accelerometers were removed at the end of the 25 minutes. At that time, participants recorded MVPA readouts on the data sheet including the time of day at which the MVPA was recorded. At a later point, the lead researcher uploaded data from the GT3X+ accelerometers focusing upon the time periods recorded by the participants.

\section{Data analysis}

Agreement was analyzed between self-counted steps and pedometer-recorded steps to examine the accuracy of the GFSP for use as a pedometer during guided walking. In addition, we analyzed agreement between the accelerometers and MVPA readouts on the GFSP to explore the accuracy of the device in measuring MVPA in the physical fitness course. Mean absolute percent error (MAPE), Bland-Altman plots, and paired samples $t$-tests were utilized to evaluate agreement in both conditions.

MAPE provides a measure of the validity of each device (Holbrook, Barreira, \& Kang, 2009) with

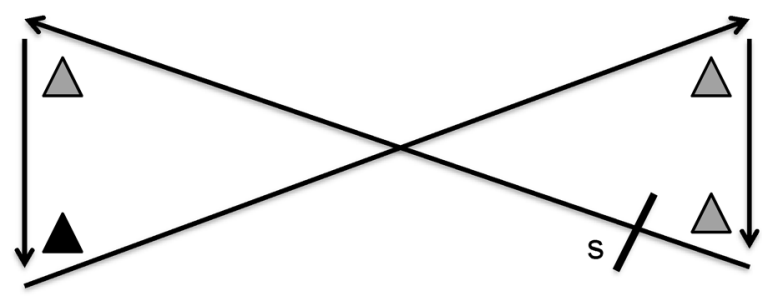

Figure 1. Closed course diagram for the two-minute test adopted from Pitchford and Yun (2011). "S" represents the starting point. 
direction of scores (positive or negative) disregarded. We calculated MAPE for guided walking ([pedometer steps-self-counted steps]/self-counted steps $\times 100)$ and free-living ([pedometer MVPA-accelerometer MVPA]/ accelerometer MVPA $\times 100$ ). Then paired samples $t$-tests were conducted for steps taken between left side and right side GFSP, left side GFSP and self-counts, as well as right side GFSP and self-counts.

We calculated Bland-Altman plots by plotting the difference between two measures (e.g. self-counts right side; accelerometer - right side) on the y-axis against the mean of the two measures on the $\mathrm{x}$-axis (e.g. self-counts + right side/2; accelerometer + right side/2). Upper ([SD of the difference $\times 1.96]+$ Mean of the difference) and lower ([SD of the difference $\times-1.96]$ + Mean of the difference). $95 \%$ confidence limits were placed on each plot as well as the mean of the difference between the two measures within each plot (middle line). Bland-Altman plots provide a graphical description of the agreement between forms of measurements and determine their interchangeability (Bland \& Altman, 1986). All statistical procedures were conducted using IBM SPSS software (Version 22.0; IBM, Armonk, NY, USA) with a significance level of alpha $\leq .05$.

\section{Results}

\section{Guided walking}

Participants completed the training video test at 95 to $100 \%$ accuracy $(M=98 \% ; S D=1.5 \%)$. During phase one, GFSP actual step counts for the right side ranged from 178 to 241 per session $(M=210)$ and for the left side ranged from 180 to $240(M=211)$. Selfstep counts ranged from 178 to $240(M=209)$. Right side pedometer MAPE with self-step counts was $1.7 \%$ $(S D=2.0 \%)$. Left side pedometer MAPE with self-step counts was $1.9 \%(S D=2.1 \%)$. The results of the paired samples $t$-tests indicate that the difference between right side and left side GFSPs was not significantly different than zero $(p=.138$; Table 1$)$. The difference between the right side GFSP and self-step counters was also not significantly different than zero $(p=.084$; Table 1). However, the difference between the left side pedometer and self-step counts was significantly different than zero ( $p=.006$; Table 1). Bland-Altman plots (Figure 2) indicated agreement with no proportional bias for the right side against self-counts and between the right and left sides. However, there is slight proportional bias between left side against self-counts.

\section{MVPA}

MVPA for the right side GFSP ranged from .5 to 19.8 minutes per session $(M=10.3)$ and for the left side GFSP ranged from .2 to 15.5 minutes $(M=7.6)$. MVPA for the accelerometer ranged from 6 to 19.8 minutes $(M=14.8)$. Right side GFSP and accelerometer MAPE was $41 \%(S D=24 \%)$ and left side GFSP with accelerometer was $51 \%(S D=22 \%)$. The results of the paired samples $t$-tests indicate that the difference between right side and left side GFSP MVPA was significantly different than zero $(p<.001$; Table 2$)$, that the difference between right side GFSP MVPA and the accelerometer was significantly different than zero $(p<.001$; Table 2), and that the difference between left side GFSP MVPA and the accelerometer was significantly different than zero $(p<.001$; Table 2$)$. BlandAltman plots indicated no proportional bias but high variability for the right side against the accelerometer, the left side against the accelerometer (Figure 2) and between the right and left side GFSPs (Figure 2).

\section{Discussion}

The purpose of this study was to evaluate the accuracy of the GFSP for measuring steps taken and MVPA across two conditions. This study produced varied results depending on the function of the instrument. The GFSP displayed MAPE in the guided walking condition of $1.7 \%$ (right side) and $1.9 \%$ (left side) that fall within the acceptable range of $3 \%$ error used in

Table 1

Results for paired samples t-tests at two minutes for step counts

\begin{tabular}{lccc}
\hline & Diff & $t$ & $p$ \\
\hline R vs. self & $-1.57 \pm 5.23$ & 1.78 & .084 \\
L vs. self & $-2.60 \pm 5.28$ & -2.92 & .006 \\
L vs. R & $-1.03 \pm 4.01$ & -2.41 & .138 \\
\hline
\end{tabular}

Note. $\quad$ Diff $=$ difference between measures; $\mathrm{R}=$ right side pedometer; $\mathrm{L}=$ left side pedometer; self $=$ self-counts.

Table 2

Results for paired samples t-tests for MVPA minutes

\begin{tabular}{lccc}
\hline & Diff & $t$ & $p$ \\
\hline R vs. L & $-2.64 \pm 3.31$ & -9.95 & $<.001$ \\
R vs. A & $-4.57 \pm 5.17$ & -5.23 & $<.001$ \\
L vs. A & $-7.21 \pm 4.29$ & -4.72 & $<.001$ \\
\hline
\end{tabular}

Note. Diff $=$ difference between measures; $\mathrm{R}=$ right side pedometer; $\mathrm{L}=$ left side pedometer; $\mathrm{A}$ = accelerometer. 

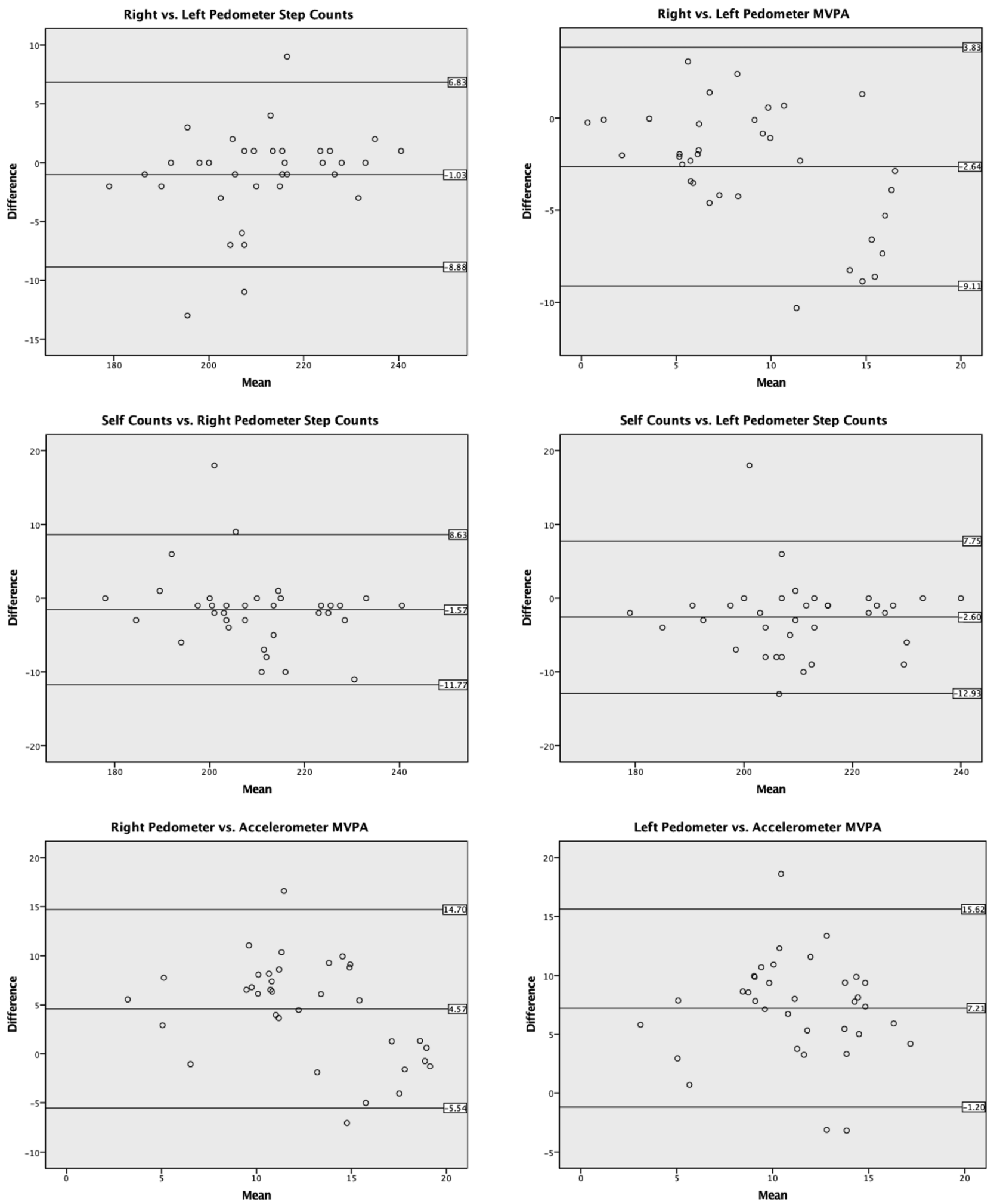

Figure 2. Bland-Altman plots for agreement of step counts and MVPA.

previous pedometer validation research in clinical settings (Schneider, Crouter, Lukajic, \& Bassett, 2003). Results from Bland-Altman plots and inferential statistics provide further support of the accuracy of the GFSP when counting steps. While the MAPE for all step counts fell within the acceptable range, placement on the right side of the body contained a slightly smaller MAPE than placement on the left side of the body when measuring steps.

The results of this study have some important implications. For physical educators, pedometers are commonly recommended and used to evaluate and promote 
physical activity in classes (Brusseau \& Burns, 2015). Unfortunately, though, many low-cost pedometers have limited capacities, particularly for saving data, placing limitations on their utilization in physical education classes. For example, one commonly used pedometer in physical activity research, the Yamax Digiwalker SW-200, is considered the "gold standard" for pedometer accuracy (Brusseau et al., 2011). However, this pedometer does not have data saving or organizing capacities, limiting physical educators to recording data after each consecutively counted session or relying on participants to self-record. Other pedometers that do have built-in memories, such as the Accusplit AH120, are either higher priced or have questionable accuracy (Trapp et al., 2013). The GFSP, on the other hand, can be uploaded to a computer to save step count data over time and organize activity by time, allowing physical educators a more convenient and easy to use way to collect data during classes.

Unlike data regarding steps taken, the results of this study suggest that the GFSP was less accurate in measuring MVPA. More specifically, large MAPE (41\% and 51\%) and high variability demonstrated with the Bland-Altman plots suggest that the GFSP may not be a suitable instrument for accurately measuring MVPA. This result is somewhat disappointing, as an affordable device that accurately measures MVPA could be a valuable resource for physical educators promoting physical activity. These results provide support for the utilization of higher priced accelerometers when measuring MVPA, which are typically reserved for research purposes. Accelerometers, such as the $\mathrm{GT} 3 \mathrm{X}^{+}$produced by ActiGraph, are known entities which have been evaluated for reliability and validity across various populations, including children (Hänggi, Phillips, \& Rowlands, 2013), adults (Jarrett et al., 2015), and those with disabilities (O'Neil, Fragala-Pinkham, Forman, \& Trost, 2014). Although a lower priced alternative could provide several benefits to physical educators, results from this study suggest that one lower priced alternative (the GFSP) would not provide adequate or comparable data to the GT3X+. Until improvements can be made to capability of these devices to accurately measure MVPA, educators should look for other options if this is the outcome variable of interest.

The importance of an early start to a physically active lifestyle has lead researchers to encourage schools to promote physical activity for all school-aged youth throughout the school-day (Metzler, McKenzie, van der Mars, Barrett-Williams, \& Ellis, 2013) and the utilization of pedometers in school-based programming is becoming more common. This includes during physical education classes, where it is becoming increasingly common for teachers to use pedometers as a measure of physical activity throughout their classes (Morgan, Pangrazi, \& Beighle, 2003). Because the GFSP offers a number of features that make utilization for physical education teachers convenient (e.g., printable reports, large display screen), and the accuracy this device has in measuring steps, this device may be a reasonable selection for school-based practices. However, this study suggests that the function that makes it unique to other low-cost physical activity monitors (measuring MVPA) is not accurate. For product consumers, including physical education personnel, utilizing a device which significantly underestimates physical activity may act as a deterrent, rather than a facilitator, to future activity. A number of studies have been conducted describing the motivational aspect of using personal physical activity monitor technology (Kang, Marshall, Barreira, \& Lee, 2009). Objective physical activity monitors may become less motivational, however, if users participate in a substantial amount of physical activity and those levels are significantly underestimated. In this instance, participants would not gain the positive reinforcement necessary from the physical activity monitors to continue to be physically active.

\section{Limitations}

The present study had several limitations that warrant consideration. First, in the third phase of the study (measuring MVPA accuracy), participants were asked to participate in two MVPA physical fitness activities that include intermittent activity patterns. Accordingly, future research is needed to evaluate the GFSP pedometer for measuring MVPA in a closed setting prior to any further applied research. Second, the researchers did not examine the accuracy of the GFSP in measuring MVPA in the guided walking setting prior to moving onto the MVPA physical fitness condition. This omission was made because the guided pace selected for the guided walking condition would have been below the moderate-intensity threshold. Therefore, agreement may have been inflated between the GFSP and accelerometer because MVPA counts would have been zero. Third, although common practice in evaluating the accuracy of objective measures is to compare new instruments to previously validated ones (such as the ActiGraph GT3X+ in this study), future research may consider the use of a systematic observation of physical activity to include multiple comparable measures and increase the rigor of the study. Fourth, the current study utilized college-aged participants. While this, in and of itself, is not a limitation, consumers should be cautious when applying the results of this study to school-aged populations. 
This device in particular has been marketed heavily toward physical education teachers as a convenient device that is low-cost and provides physical activity outputs. Because this study was conducted on adults, further research would be necessary to ensure that the GFSP accuracy measures steps for younger schoolaged children with shorter gaits. Next, the current study utilized a self-counting method for the criterion for evaluation of step count accuracy. Although participants completed self-count training prior to data collection, there is always a possibility for participants to lose count of their steps or lie about their step count. A number of protocols were put into place to eliminate that potential, including using short bouts (i.e., two minutes), covering the instruments display screens while walking, and asking participants to record their counted steps prior to opening the display screen to record pedometer recorded steps. Finally, the manner through which each device (ActiGraph and GFSP) assesses MVPA differs. The accelerometer measures MVPA based upon intensity above a cut point. The GFSP measures MVPA based upon a ratio of steps per minute above a self-selected ratio for walking steps per minute. Moreover, various settings within the ActiGraph can influence outputs (e.g., length of epoch, chosen cut points, use of filters, etc.). Research varies upon which settings to use with ActiGraph accelerometers. As such, results should be interpreted with caution.

\section{Conclusions}

A low-cost physical activity monitor that can measure both steps taken and MVPA could be a valuable measurement tool for those unable to purchase high cost activity monitors (e.g., schools) or those interested in multiple outcome variables (e.g., steps taken and MVPA). The GFSP is one such monitor that has these capabilities and has been heavily marketed as a tool that is affordable and easy to use for physical educators. However, the results of this study suggest that although the GFSP can measure steps taken accurately in a guided walking environment, the device significantly underestimates MVPA. Currently on the market, there are numerous pedometers that measure steps taken accurately which can be utilized in schools and are less expensive than the GFSP. Thus, because the GFSP significantly underestimates MVPA and it would not be considered a cost-effective measure of steps taken, it may not be the most practical suggestion for utilization in physical education.

\section{Acknowledgments}

The authors wish to thank Drs. Jackie Goodway, YuChun "Jean" Chen, Tammy Schilling, and Lanie Dornier for their assistance with this study.

\section{Conflict of interest}

There were no conflicts of interest.

\section{References}

Barreira, T. V., Tudor-Locke, C., Champagne, C. M., Broyles, S. T., Johnson, W. D., \& Katzmarzyk, P. T. (2013). Comparison of GT3X accelerometer and Yamax pedometer steps/days in a free-living sample of overweight and obese adults. Journal of Physical Activity \& Health, 10, 263-270.

Bland, J. M., \& Altman, D. (1986). Statistical methods for assessing agreement between two methods of clinical measurement. Lancet, 327, 307-310.

Brusseau, T. A., \& Burns, R. D. (2013). Pedometer-determined physical activity of youth while attending school: A review. Sport Science Review, 22, 329-342.

Brusseau, T. A., \& Burns, R. D. (2015). Step count and MVPA compendium for middle school physical education activities. Journal of Physical Education and Sport, 15, 646-650.

Brusseau, T., Kulinna, P. H., Tudor-Locke, C., Ferry, M., van der Mars, H., \& Darst, P. W. (2011). Pedometer-determined segmented physical activity patterns of fourth- and fifth-grade children. Journal of Physical Activity \& Health, 8, 279-286.

Clemes, S. A., \& Biddle, S. J. H. (2013). The use of pedometers for monitoring physical activity in children and adolescents: Measurement considerations. Journal of Physical Activity \& Health, 10, 249-262.

Crouter, S. E., Schenieder, P. L., \& Bassett, D. R. (2005). Spring-levered versus piezo-electric pedometer accuracy in overweight and obese adults. Medicine \& Science in Sports \& Exercise, 37, 1673-1679.

Elies, S. (2015). Performance analysis of commercial accelerometers: A parameter review. Sensors \& Transducers, 193, 179-190.

Freedson, J., Melanson, E., \& Sirard, J. (1998). Calibration of the Computer Science and Applications, Inc. accelerometer. Medicine \& Science in Sport \& Exercise, 30, 777-781.

Gatti, A., Stratford, P., Brenneman, E., \& Maly, M. (2015). GT3X+ accelerometer placement affects the reliability of step-counts measured during running and pedal-revolution counts measured during bicycling. Journal of Sports Sciences, 33, 1-8.

Gopher. (2015). FITStep Pro uploadable pedometers. Retrieved from http://www.gophersport.com/ pe/teacher-resources-pedometers/FITStep-pro-uploadablepedometers?ca $=1809$

Hänggi, J. M., Phillips, L. R., \& Rowlands, A. V. (2013). Validation of the GT3X ActiGraph in children and 
comparison with the GT1M ActiGraph. Journal of Science and Medicine in Sport, 16, 40-44.

Hasson, R. E., Haller, J., Pober, D. M., Staudenmayer, J., \& Freedson, P. S. (2009). Validity of the Omron HJ-112 pedometer during treadmill walking. Medicine \& Science in Sport \& Exercise, 41, 805-809.

Holbrook, E., Barreira, T. V., \& Kang, M. (2009). Validity and reliability of Omron pedometers for prescribed and self-paced walking. Medicine \& Science in Sports \& Exercise, 41, 670.

Jarrett, H., Fitzgerald, L., \& Routen, A. C. (2015). Interinstrument reliability of the ActiGraph GT3X+ ambulatory activity monitor during free-living conditions in adults. Journal of Physical Activity \& Health, 12, 382-387.

Kang, M., Marshall, S. J., Barreira, T. V., \& Lee, J.-O. (2009). Effect of pedometer-based physical activity interventions: A meta-analysis. Research Quarterly for Exercise and Sport, 80, 648-655.

Kurti, A. N., \& Dallery, J. (2013). Internet-based contingency management increases walking in sedentary adults. Journal of Applied Behavior Analysis, 46, 568-581.

Lee, J. A., Williams, S. M., Brown, D. D., \& Laurson, K. R. (2015). Concurrent validation of the Actigraph gt $3 \mathrm{x}^{+}$, Polar Active accelerometer, Omron HJ-720 and Yamax Digiwalker SW-701 pedometer step counts in lab-based free living settings. Journal of Sports Sciences, 33, 991-1000.

Metzler, M., McKenzie, T. L., van der Mars, H., Barrett-Williams, S. L., \& Ellis, R. (2013). Health optimizing physical education: A new curriculum for school programs - Part 1: Establishing the need and describing the model. Journal of Physical Education, Recreation, \& Dance, 84(4), 23-28.

Morgan, C. F., Pangrazi, R. P., \& Beighle, A. (2003). Using pedometers to promote physical activity in physical education. Journal of Physical Education, Recreation, \& Dance, 74(7), 33-38.

O’Neil, M. E., Fragala-Pinkham, M. A., Forman, J. L., \& Trost, S. G. (2014). Measuring reliability and validity of the ActiGraph GT3X accelerometer for children with cerebral palsy: A feasibility study. Journal of Pediatric Rehabilitation Medicine, 7, 233-240.

Pangrazi, R. P., Beighle, A., \& Sidman, C. L. (2003). Pedometer power: 67 lessons for K-12. Champaign, IL: Human Kinetics.

Pitchford, E. A., \& Yun, J. (2011). Pedometer variance in adults with Down syndrome during free walking: A generalizability study. Journal of Physical Activity \& Health, 8, 1143-1151.

Schneider, P. L., Crouter, S. E., Lukajic, O., \& Bassett, D. R. (2003). Accuracy and reliability of 10 pedometers for measuring steps over a 400-m walk. Medicine \& Science in Sports \& Exercise, 35, 1779-1784.

Steeves, J. A., Tyo, B. M., Connolly, C. P., Gregory, D. A., Stark, N. A., \& Bassett, D. R. (2011). Validity and reliability of the Omron HJ-303 tri-axial accelerometerbased pedometer. Journal of Physical Activity \& Health, 8, 1014-1020.

Trapp, G. S. A., Giles-Corti, B., Bulsara, M., Christian, H. E., Timperio, A. F., McCormack, G. R., \& Villanueva, K. (2013). Measurement of children's physical activity using a pedometer with a built-in memory. Journal of Science and Medicine in Sport, 16, 222-226.

Tudor-Locke, C., \& Myers, A. M. (2001). Methodological considerations for researchers and practitioners using pedometers to measure physical (ambulatory) activity. Research Quarterly for Exercise and Sport, 72, 1-12.

Wetten, A. A., Batterham, M., Tan, S. Y., \& Tapsell, L. (2014). Relative validity of 3 accelerometer models for estimating energy expenditure during light activity. Journal of Physical Activity \& Health, 11, 638-647.

Yang, C.-C., \& Hsu, Y.-L. (2010). A review of accelerometry-based wearable motion detectors for physical activity monitoring. Sensors, 10, 7772-7788. 